\title{
Mental imagery and visual working memory abilities appear to be unrelated in childhood: evidence for individual differences in strategy use
}

\author{
Kathryn E. Bates ${ }^{1 *}$, Emily K. Farran ${ }^{2}$
}

1. Department of Psychology and Human Development, University College London Institute of Education, 25 Woburn Square, London, UK, WC1H OAA.

2. School of Psychology, University of Surrey, Guildford, Surrey, UK. GU2 7XH.

*corresponding author: kathryn.bates.16@ucl.ac.uk

The author(s) declare no competing interests.

The current version of this manuscript has been revised following reviewer comments and is currently under review with Cognitive Development. Supplementary materials can be found here: https://osf.io/rk7f5/ 
Abstract: We addressed two research gaps related to mental imagery (MI) in children. First, research has investigated the development of $\mathrm{MI}$ employing a component model (image generation, image maintenance, mental rotation, image scanning), however knowledge of how components develop and their associations throughout childhood (age 6-11 years) is incomplete. Second, despite positive associations in adults, the relationship between $\mathrm{MI}$ and visual working memory (VWM) has not been investigated in children. Using a novel MI task battery sensitive to the visual precision of MI, we found evidence for a separable-component model of $\mathrm{MI}$ in children, with some components becoming integrated in adulthood. Contrary to expectations, there were no significant associations between components of $\mathrm{MI}$ and VWM in either childhood or in adults. Our findings extend current understanding of development of $\mathrm{MI}$ and highlight the importance of considering individual differences in strategy-use when establishing the relationship between $\mathrm{MI}$ and VWM.

Keywords: Mental Imagery, Visual Working Memory, Cognitive Development, Cognitive Strategies 


\section{Highlights:}

- Mental imagery (MI) task battery suitable for both children and adults

- Components of MI develop separately and become integrated in adulthood

- No significant associations between MI and visual working memory; implies variability in strategies 


\section{Main Text:}

\section{Introduction}

Mental imagery (MI) is defined as the ability to generate an image in mind in the absence of sensory input (Kosslyn, 1994; Kosslyn, 1980). Research investigating the cognitive mechanisms of $\mathrm{Ml}$ has focused on two key aspects of MI. The first on the sensory experience of MI with the aim to determine the format of mental images. Decades of behavioural and neuroimaging research on the format of mental images in adults has provided extensive support for a depictive theory of MI. Specifically, this refers to the concept that MI can involve percept-like, visually precise representations that are distinct from the propositional or symbolic representations of general thought (Kosslyn et al., 2006; Pearson \& Kosslyn, 2015; see Pylyshyn, 2002 for discussion on propositional representations). Despite extensive research in adults, the investigation of the format of MI in developmental samples is limited. The second key avenue of research has focused on the computational model of $\mathrm{MI}$ which posits a component model consisting of image generation, image maintenance, image transformation/rotation and image scanning/inspection (see Kosslyn et al., 2006 for review). This body of research has demonstrated that MI can be differentiated from verbal and non-verbal reasoning abilities and there is evidence for separable components of Ml in adulthood (Kosslyn, 1994; Kosslyn et al., 1984). However, evidence for the relationship between components of $\mathrm{MI}$ in childhood is limited. Furthermore, both behavioural and neuroimaging literature have demonstrated a positive relationship between MI and visual working memory (VWM; Albers et al., 2013; Keogh \& Pearson, 2011, 2014) and MI and visual perception (Dijkstra et al., 2017, 2019) in adults, which has been vital in understanding how we use $\mathrm{MI}$ in memory and understanding the sensory experience of MI. While some have speculated that VWM abilities might play a role in the development of $\mathrm{MI}$ (Wimmer et al., 2016), how MI and VWM are related throughout childhood is yet to be fully investigated.

\subsection{Is MI unitary or multi-faceted?}

While one strand of research in $\mathrm{MI}$ has focused on determining the format of representations, the other has investigated the cognitive function of MI. Following the early theorisation of the sub-component model of $\mathrm{MI}$, few studies have applied this model in $\mathrm{MI}$ research. As the sensory experience of $\mathrm{MI}$ is variable, research has thus far largely focused on using neuroimaging techniques to uncover the format of representations recruited in $\mathrm{MI}$, which has revealed evidence for visually depictive mental images. However, this neglects the wealth of information that can be provided by investigating psychological phenomena using carefully crafted behavioural tasks. While the binocular rivalry paradigm has been 
adopted in $\mathrm{Ml}$ research as a behavioural measure of the sensory strength of $\mathrm{MI}$ (Pearson et al., 2008; Pearson \& Kosslyn, 2015) this does not account for the complexity of MI abilities. A key observation in the early development of a theoretical model of $\mathrm{MI}$ was that $\mathrm{MI}$ is not a unitary construct: rather, it is comprised of multiple sub-abilities that are distinct from one another and influenced by extensive individual variation (Kosslyn, 1980; Kosslyn et al., 2006). Such a definition is more akin to the way in which we experience $\mathrm{Ml}$ in everyday life, i.e., a highly visual image is not always the most efficient. For example, a highly visual image might be useful when trying to visualise a descriptive scene whilst reading a story. On the other hand, envisioning how your luggage might fit in the boot of a car is likely more dependent on your ability to transform spatial relations in mind. Some abilities might be dependent on both strong visual and spatial features, such as describing the best route when giving directions. Nevertheless, research adopting a sub-component model to investigate the mechanisms of $\mathrm{Ml}$ is limited.

Establishing whether or not MI can be conceptualised as a multi-faceted function has important implications for examining how components of MI support other high-order cognitive functions, such as VWM, and learning outcomes. While the direct assessment of how each of the components of MI relate to one another is limited, some research in the MI literature has examined how MI abilities contribute to different types of reasoning and problem-solving. Firstly, in adults, relationships have been demonstrated between image scanning abilities and spatial transformation tasks, such as folding. Namely, image scanning RT slopes and accuracy were found to positively correlate with paper folding but did not correlate with scores on the object-imagery scale on the Object-Spatial Imagery Questionnaire (OSIQ) (Blajenkova et al., 2006; Borst \& Kosslyn, 2010). The OSIQ includes items referring to the visual properties and precision of images, e.g., "My mental pictures are very precise representations of real things". Thus, this preliminary evidence suggests a distinction between ability in representing spatial properties in mental images compared to the visual precision of mental images. This notion is supported by recent studies of individuals with no visual imagery; recently described as individuals with Aphantasia (Zeman et al., 2015). While presenting with poor or absent ability to generate visual images, those with Aphantasia appear to score in the typical range on the spatial imagery scale of the OSIQ, which includes items such as "I can easily rotate 3D geometric figures" (Keogh \& Pearson, 2017). These findings are limited to self-report data, however a recent study showed mental rotation performance in those with Aphantasia is on par with controls (Pounder et al., 2021). These initial findings point towards a dissociation between the visual precision of images and spatial properties of images in adults. With respect to development of MI throughout childhood, it was initially argued that while MI plays a role in children's 
thinking, $\mathrm{MI}$ in children younger than 7 years is restricted to the generation and maintenance of static images, while the ability to transform images develops later (Bruner, 1966; Piaget \& Inhelder, 1971). This pertains to the argument above in the adult literature regarding a possible distinction between visual and spatial aspects of MI. Research has since demonstrated the presence of mental rotation ability in as early as 3 months old (Hespos \& Rochat, 1997; Moore \& Johnson, 2011), yet the ability to rotate and transform depictive images has been found to undergo extensive development between the ages of 4 and 6 years (Frick et al., 2013; Wimmer et al., 2017). Alongside this, earlier research suggested image generation ability develops into later childhood compared to image maintenance (Kosslyn et al., 1990) and while this was supported in a recent study, it was also found that both image generation and image maintenance abilities were present in children as young as 4 years (Wimmer et al., 2017). Overall, further clarity is needed on the developmental progression of each component of $\mathrm{Ml}$ and how visual and spatial components relate to one another throughout development.

To date, Kosslyn et al.'s (1990) study is the only study of MI in children to compare all four components throughout development and in adulthood. This study demonstrated support for a separable-component model in that, with the exception of a positive relationship between error rates in mental rotation and image scanning at age 8 , the four components were statistically unrelated to one another for all age groups. Notably, the age groups in Kosslyn et al. (1990) were 5-year-olds, 8-year-olds and 14-year-olds, therefore there is a gap in knowledge regarding the relationship between components of $\mathrm{Ml}$ in mid to late childhood. More recent developmental research is mixed and pairs of components, i.e., image generation and image maintenance (Wimmer et al., 2015) and mental rotation and image scanning (Wimmer et al., 2017), have been compared in separate studies. Firstly, accuracy was higher in image maintenance compared to image generation in childhood, which was argued to suggest image generation involves additional processes to image maintenance (Wimmer et al., 2015), and the association between the two components was not examined. Second, with regards to mental rotation and image scanning, there were no significant associations when age was controlled for, however 6-, 10-year-olds and adults, but not 8-year-olds, showed significant positive relationships between mental rotation and image scanning (Wimmer et al., 2017). Using tasks designed to capture how visually depictive representations are recruited in each of the components of $\mathrm{MI}$ in children aged 611 years, this paper will provide important clarifications on whether how components of $\mathrm{MI}$ are related throughout childhood.

To establish the relationship between components throughout childhood, tasks are required that tap into the visual precision on $\mathrm{MI}$, i.e., the extent to which a depictive theory of 
MI is supported throughout childhood. This will provide important clarifications as to the format of $\mathrm{MI}$ in children. The suggestion that RT increases as a function of degrees of rotation and distance scanned in mental rotation and image scanning, respectively, suggests depictive representations are recruited in these tasks (Borst \& Kosslyn, 2010; Shepard \& Metzler, 1971). Support for a depictive theory of MI in adults is derived largely from neuroimaging findings evidencing shared neural activation between $\mathrm{Ml}$ and visual perception. Namely, there is strong evidence of corresponding activity in the primary visual cortex (V1) during MI (Dijkstra, Bosch, \& van Gerven, 2019; Ganis, 2013; Kosslyn, Ganis, \& Thompson, 2003; Kosslyn \& Thompson, 2003). Because V1 is retinotopically organised so that actual distance coordinates in space are mapped to corresponding coordinates on the cortex, neural activation in this area supports the suggestion for depictive representations in MI. Recent evidence demonstrating that activation in V1 during MI can be decoded using classifiers trained on activation in visual perception further supports the argument that visual images are generated in Ml (Albers et al., 2013; Naselaris et al., 2015). Thus, the cumulative evidence strongly supports the role of depictive representations of $\mathrm{MI}$, as well as individual differences in the precision of visual images in adults. While there is now little contestation of Kosslyn's depictive theory in the literature, this is limited to adult populations and the extent to which a depictive theory of $\mathrm{MI}$ is supported across childhood represents a gap in the literature that the present study addresses.

To summarise, it is currently unclear how the ability to transform an image (mental rotation) and represent distances within an image (image scanning) relates to the ability to generate and maintain visual images (image generation and image maintenance, respectively) throughout development in primary years and in adulthood. To address this issue, we will investigate the associations between each component of MI to directly assess how abilities in each of the subcomponents of MI are related throughout childhood.

\subsection{The relationship between MI and VWM}

Central to our understanding of the function of $\mathrm{MI}$ and the format of representations in $\mathrm{MI}$ is the investigation of how MI relates to VWM ability. The functions of MI seem to emulate those of VWM in that both theories of WM and theories of MI suggest that visual information can be stored temporarily, focussed on at will and reactivated as needed (Cowan, 2001; Kosslyn et al., 2006; Logie, 1995). In a paradigm designed to assess the sensory strength of visual images (i.e., the greater the bias to perceive an imagined stimulus in subsequent perception of that stimulus, the greater the sensory strength of an image), behavioural studies have found a positive relationship between MI and VWM capacity. Importantly, this relationship was not found between non-VWM abilities such as iconic and numeric working 
memory (Keogh \& Pearson, 2011), thus suggesting that the recruitment of visual images in VWM is associated with better performance. Moreover, a study introducing irrelevant visual information to try and disrupt performance in VWM found that only individuals who scored highly on the sensory strength measure of MI were disrupted (Keogh \& Pearson, 2014), thus suggesting that variance in strategy use during VWM may be related to individual differences in MI ability. In addition, there is evidence for shared neural representations in MI and VWM. It has been shown that neural activation associated with VWM in the early visual areas can be decoded by classifiers trained on activation during MI trials and vice versa (Albers et al., 2013). This cumulative evidence has led to the argument that individual differences in VWM may be dependent on the format of representations and whether MI strategies are employed in VWM tasks (Pearson \& Keogh, 2019; Reeder, 2017). Therefore, examining which components of $\mathrm{MI}$ are related to VWM performance throughout development will further elucidate how MI underpins individual differences in VWM and whether this varies over developmental time.

While it has been speculated that variation in the involvement of VWM might play a role in the relationship between components of MI (Wimmer et al., 2017), developmental studies are yet to directly examine the relationship between $\mathrm{MI}$ abilities and VWM. The development of VWM, and how this function might aid learning and academic achievement, has been investigated extensively and has largely demonstrated a positive relationship between VWM abilities and academic skills, such as mathematics and reading comprehension (e.g. Berg, 2008; Bull, Espy, \& Wiebe, 2008; Giofrè et al., 2013). Despite this, training VWM appears to have little effect on academic abilities (Sala \& Gobet, 2017). MI has also been found to be positively associated with reading skills (e.g. Commodari, Guarnera, Di Stefano, \& Di Nuovo, 2020) and there is evidence to suggest that visualisation abilities underpin mathematics ability (Bates et al., 2021; Hawes et al., 2019; Hegarty \& Kozhevnikov, 1999). Moreover, studies with university students have demonstrated that encouraging MI strategies can aid learning of scientific concepts (Leopold et al., 2015). Taken together, the evidence suggests further understanding of the development of MI alongside VWM could have vital implications for learning strategies in the classroom.

\subsection{The current study}

The current study was designed to investigate how components of $\mathrm{MI}$ are related and how components of $\mathrm{MI}$ are associated with VWM throughout development in primary school years and in adulthood. We have developed a MI battery, designed to be suitable from 6 years to adulthood. While we acknowledge previous studies have found the presence of MI abilities from age 4, required amendments made to the paradigm to assess the visual 
precision of mental images rendered the current battery of tasks too difficult for children under 6 years. To examine adult-like abilities and assess the extent to which abilities differentiate over time it was vital to use measures that were also sensitive to adult-levels of visual precision.

The image generation and image maintenance tasks employed here were adapted from Wimmer et al. (2015). The paradigm follows the same sequence as Wimmer et al. (2015), however we used abstract shapes instead of known stimuli (e.g., flowers). Based on Wimmer et al.'s (2015) study, the current image generation task involved a 30 second distractor task to encourage the visual image to be generated from long-term memory, whereas the image maintenance task involved a 3 second delay to encourage the visual image to be maintained. While it has been argued that known stimuli are easier to visualise (e.g. Kail, Pellegrino, \& Carter, 1980), the use of known stimuli encourages verbalising strategies, whereas verbalising is a less efficient strategy for abstract shapes. As such, in the current study, participants are required to memorise an abstract shape and detect the location of a difference in the target shape. Participants must first distinguish whether there is a difference in the target shape, and if there is a difference, they then have to locate the exact difference, which allows us to categorically distinguish between generating and maintaining visual images with high precision and low precision, respectively. We expect faster response times (RTs) for high precision responses compared to low precision responses. This would demonstrate that in high precision responses, participants are referring to a visual image of high precision, whereas in low precision responses, participants struggle to detect the difference using a visual image and therefore recruit a less efficient, non-imagery strategy such as verbalising.

These novel tasks were paired with a mental rotation task (as used in Gilligan, Thomas, \& Farran, 2019; adapted from Neuburger et al., 2011) and an image scanning task modified from Wimmer et al. (2016). Given the extensive literature examining mental rotation, we expect to replicate the robust finding that as degree of rotation increases, RT increases, thus implying that individuals are rotating an image in mind in this task and supporting evidence for depictive representations. Similarly, in image scanning, we expect RTs to increase as a function of distance scanned (Wimmer et al., 2016). To examine individual differences in visual precision of mental images in image scanning, we will assess how mentally represented distance corresponds to actual distance perceived in perception control trials. We will investigate this by deriving a measure of distance represented from RT. The logic for this measure is based on the same logic that has been paramount to research investigating mechanisms of mental rotation; namely, the established finding that RT increases as a function of distance rotated. Given that previous research has shown RT increases as a 
function of distance scanned in image scanning tasks, it is reasonable to assume that we can use response times to estimate participants' ability to represent varying distances in mind. If mental images of visual precision are recruited, we predict that individuals will show similar patterns of error in imaged distance compared to perceived distance.

The key contributions of this paper are to examine: 1) the developmental progression of the components of $\mathrm{MI}$ throughout primary school years, 2) the relationship between components of $\mathrm{MI}$ and 3 ) the relationship between each component and VWM. With reference to point 1 , we predict improvement in the visual precision of image generation and image maintenance throughout childhood. Given the mixed results in the previous research and the fact that our tasks tap into the visual precision of $\mathrm{MI}$, we do not have any predefined hypotheses regarding the age at which adult-like abilities will be evident. With reference to point 2, we predict a relationship between visual components (i.e., image generation and image maintenance) and an association between the components involving transformations (mental rotation and image scanning). With reference to point 3, while the relationship between components of $\mathrm{MI}$ and VWM are yet to be investigated in childhood, the evidence for a positive relationship between $\mathrm{MI}$ and VWM in adults is argued to be dependent on the recruitment of visual representations in both functions (e.g., Keogh \& Pearson, 2014). The VWM tasks presented here comprise of a forward span and backward span VWM task. The choice to include both measures is in line with suggestions that forward span represent maintenance in VWM and backward span represents manipulation in VWM (e.g. Bull, Espy, \& Wiebe, 2008; Martinussen \& Tannock, 2006). Therefore, we can compare children's abilities in MI components to the ability maintain a visual representation in VWM and manipulate a representation in VWM for the first time. Thus, if children are using MI strategies to support VWM performance, as suggested in the adult literature, we would expect positive relationships at least between the ability to maintain a visual image (image maintenance) and the ability to maintain in VWM (forward span), and a relationship between the ability to transform an image (mental rotation) and manipulate in VWM (backward span).

\section{Methods}

\subsection{Participants}

Adult participants were recruited via social media and SONA recruitment systems at Birkbeck, University of London. Participants recruited via the university system were rewarded course credit and all participants were entered into a prize draw to win a $£ 50$ Amazon voucher. Children were recruited from two primary schools and received a "Young Scientist" certificate for participation. The final sample consisted of 92 children aged 6-11 years and 58 adults. The sample size suggested by a priori power analyses was 88 . Power 
was set at 0.80 , alpha level was set at 0.05 and effect size estimates ranged from small to large, dependent on the analyses, based on previous findings (Wimmer et al., 2015; 2016; 2017). Data was collected in conjunction with a larger project that involved 92 primary school children age 6-11 years and 58 adults, therefore all 92 children and 58 adults were included in the analyses below to maximise power. One participant from the adult group was excluded from the correlation analysis due to multiple mouse control errors at the start of the Backward VWM span task leading to the task stopping after only 4 trials. The total sample for these analyses included 92 children plus the 57 adult participants (total sample: $\mathrm{N}=149$ ). Participant details are in Table 1.

All adults provided written informed consent. Parents of child participants provided written informed consent and subsequently the study information was explained to the child and each child gave written informed consent. The study was approved by the University Ethics Committee and was conducted in accordance with the Declaration of Helsinki.

Table 1

Demographics for sample

\begin{tabular}{lll}
\hline Age group & $\mathrm{N}$ (female) & Age in years; months (Mean[SD]) \\
\hline 6-7-year-olds & $31(20)$ & $6 ; 11(0 ; 6)$ \\
8-9-year-olds & $26(16)$ & $8 ; 6(0 ; 6)$ \\
10-11-year-olds & $35(14)$ & $10 ; 7(0 ; 5)$ \\
Adults & $58(39)$ & $26 ; 11(6 ; 8)$ \\
\hline
\end{tabular}

\subsection{Material and procedure}

All tasks were comprised and presented on a 23-inch ASUS LCD monitor running with MATLAB v2018a (The MathWorks Inc, Natick, MA) and PsychToolbox v3.0.14. Adults participated in a single 90 -minute testing session in a quiet testing booth at the university and the order of tasks completed by each participant was counterbalanced. Children were tested at school whereby the 90 -minute session was split into 3 approximately 30 -minute sessions to minimise impact on the school day and participant fatigue. Instructions for all tasks were read by the researcher and written test instructions were presented on screen at the start of the task.

\subsubsection{Image generation and image maintenance}

Stimuli consisted of 4-sided abstract shapes with either 1 or 2 curved lines and 3 or 2 straight lines. All stimuli covered an approximately equal surface area within a space of 
$200 \times 180$ pixels. Participants were seated at a viewing distance of $57 \mathrm{~cm}$, from which images subtended a visual angle of $5.1^{\circ}$ (height) by $4.7^{\circ}$ (width).

At the beginning of each trial in the image generation task, an abstract shape was presented for 15 seconds and participants were instructed to try and remember the details of the shape. When the shape disappeared, a checkerboard mask was flashed onto the full screen for $500 \mathrm{~ms}$ to remove aftereffects. Participants were then instructed to turn to a paper and pencil letter cancellation task (Massonnié et al., 2020). The task formed the distractor task whereby participants were presented with a 16x20 grid of capital letters, evenly spaced on A4 paper. Participants were required to read each line from left to right and cross out the letters $T$ and $\mathrm{G}$ ( 5 Gs and 5 Ts were interspersed randomly). After 30 seconds, a visual cue appeared on the screen and the researcher alerted the participant to turn back to the computer task. Next, they were required to think of the previous shape and identify whether the next shape to appear on the screen looked different from the reference shape. The target shape was presented, and participants were required to press the corresponding labelled letter key on the keyboard to answer either "YES" (the shape looked different) or "NO" (the shape did not look different). If the participant pressed the "YES" key, they then had to use the mouse to click on the part of the shape they thought was different, if the participant pressed the "NO" key, the task moved on to the next trial. Piloting with children showed that RTs in the image generation and image maintenance tasks were confounded by mouse control errors, therefore for the image generation and image maintenance tasks, if participants responded "YES" they then pointed with their finger to the exact location of the difference on the screen and the experimenter clicked with the mouse. This is consistent with previous methods showing no significant differences between experimenter and participant responses (Wimmer et al., 2015).

In all trials, reference and target shapes were presented in the same location. In "same" trials, the same shape was presented. In "difference" trials, the target shape presented was the reference shape with one alteration: either the concave or convex attribute of the curved line was increased or the angle between two straight lines was reduced/increased (see Figure $1 \mathrm{~A}$ ). We were careful to ensure that the trial presentation was not predictable: presentation of same and different trials was randomised per participant, the number of same and different trials were not the same (10 different trials and 5 same trials) and there were 15 different locations that each pair were presented in, therefore the location of the shape in a new trial was also not predictable.

Precision of visual images was derived from "difference" trials only, with two categories: high precision response and low precision response. High precision responses 
are responses whereby participants correctly identified a difference and the exact location of the difference, low precision responses are the responses whereby participants identified a difference but did not correctly identify the location of the difference. A dependent variable of level of precision was derived from response time: mean RT for "YES" responses to difference trials for high and low precision. RTs were calculated by summing the RT to the "YES" button press and the RT for the location mouse click. To check that low precision responses were not due to a bias to answer "YES" and subsequently guess the location of the difference, we computed one sample $t$ tests of the percentage of "YES" answers against $100 \%$, in image generation $(M=45.29, S D=13.80)$ and image maintenance $(M=52.18, S D$ $=13.94)$, respectively. The percentage of "YES" responses was significantly lower than $100 \%$ in both the image generation $(t(149)=-48.55, p<.001, d=-3.96)$ and image maintenance tasks $(t(149)=-42.02, p<.001, d=-3.43)$. Thus, we can assume that the precision categories allow us to distinguish between visual precision (high and low) of images generated and maintained in these tasks.

An overall accuracy dependent variable was calculated to investigate progression of image generation and image maintenance ability throughout primary school years and to include in the correlation analyses. High precision trials were given a score of 2 , low precision trials were given a score of 1 and incorrect responses were scored 0 . This resulted in a maximum possible overall accuracy score of 25 (10 difference trials, 5 same trials). For example, maximum score of 25 would be achieved by 10 difference trials with score of 2 (high precision) and 5 same trials with score of 1 .

The image maintenance task differed from the image generation task only in that when the shape disappeared, participants were instructed to hold the shape in mind and look at the fixation cross presented in the centre of the screen for $3000 \mathrm{~ms}$. Participants were then presented with the target shape and responded as above (see Figure 1B). Scoring was as in the image generation task. 


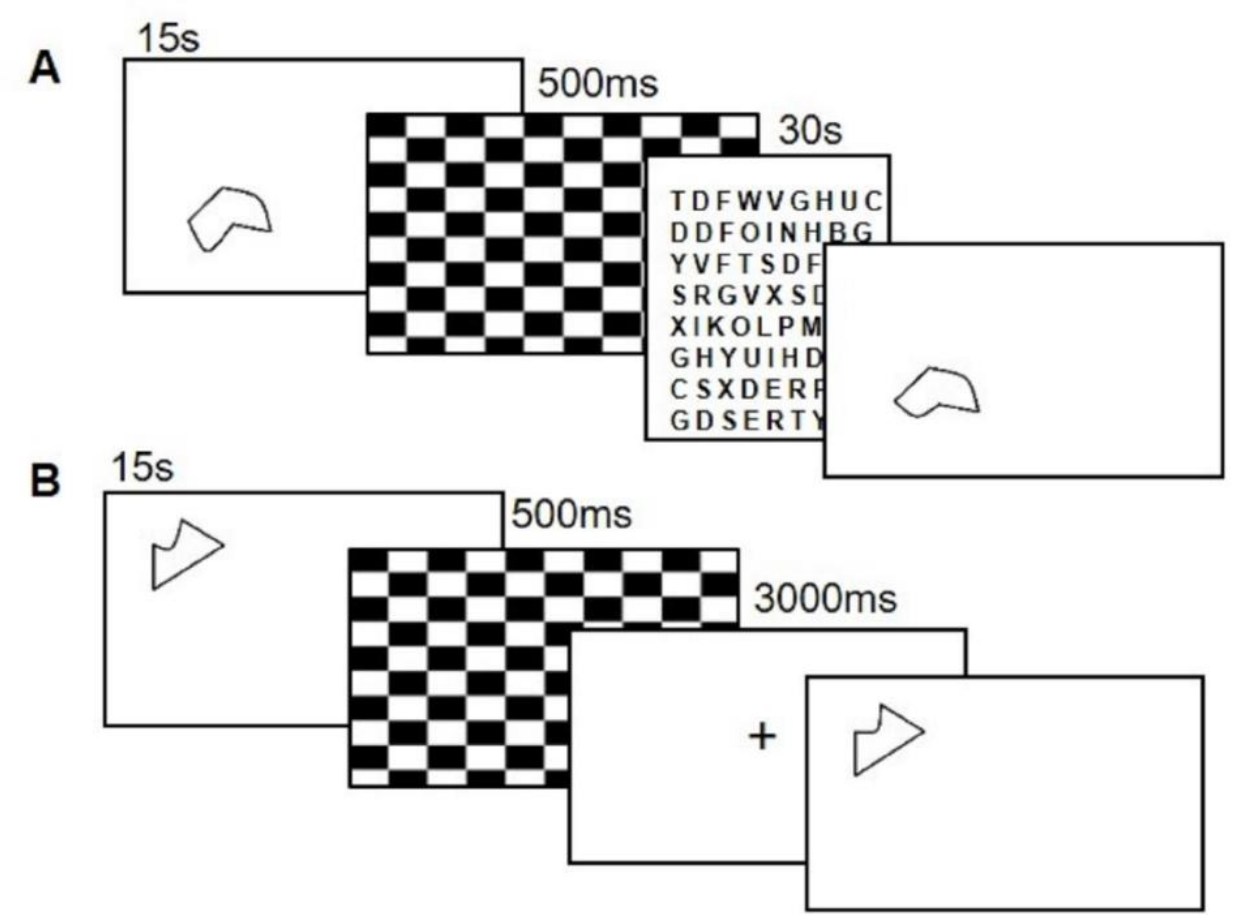

Figure 1: A) Image generation difference trial: presentation of the reference shape for $15 \mathrm{~s}$, presentation of checkerboard mask for $500 \mathrm{~ms}$, letter cancellation distractor task on A4 paper for 30s, presentation of test shape. B) Image maintenance difference trial: as above except instead of the letter cancellation task, a fixation cross appeared in the centre of the screen for $3000 \mathrm{~ms}$

\subsubsection{Mental rotation}

In the mental rotation task, participants were required to identify which of two mirrorimaged animal images located above a horizontal line, matched the target image below the line (see Gilligan et al., 2019 for details). The images above the line included a mirrored image of the target image and the target image at 0 degrees rotation (see Figure 2). Participants used the left arrow and right arrow keys on the computer keyboard to respond. Participants completed 4 practise trials at $0^{\circ}$, two with feedback. If participants scored less than $75 \%$ accuracy the practise was repeated. In experimental trials, the test image was presented at either $0^{\circ}, 45^{\circ}, 90^{\circ}, 135^{\circ}$ and $180^{\circ}$ clockwise or anti-clockwise, 8 trials per degree of rotation summing to a total of 40 trials. The order of trial presentation was randomised per participant. RTs for correct trials were recorded. 

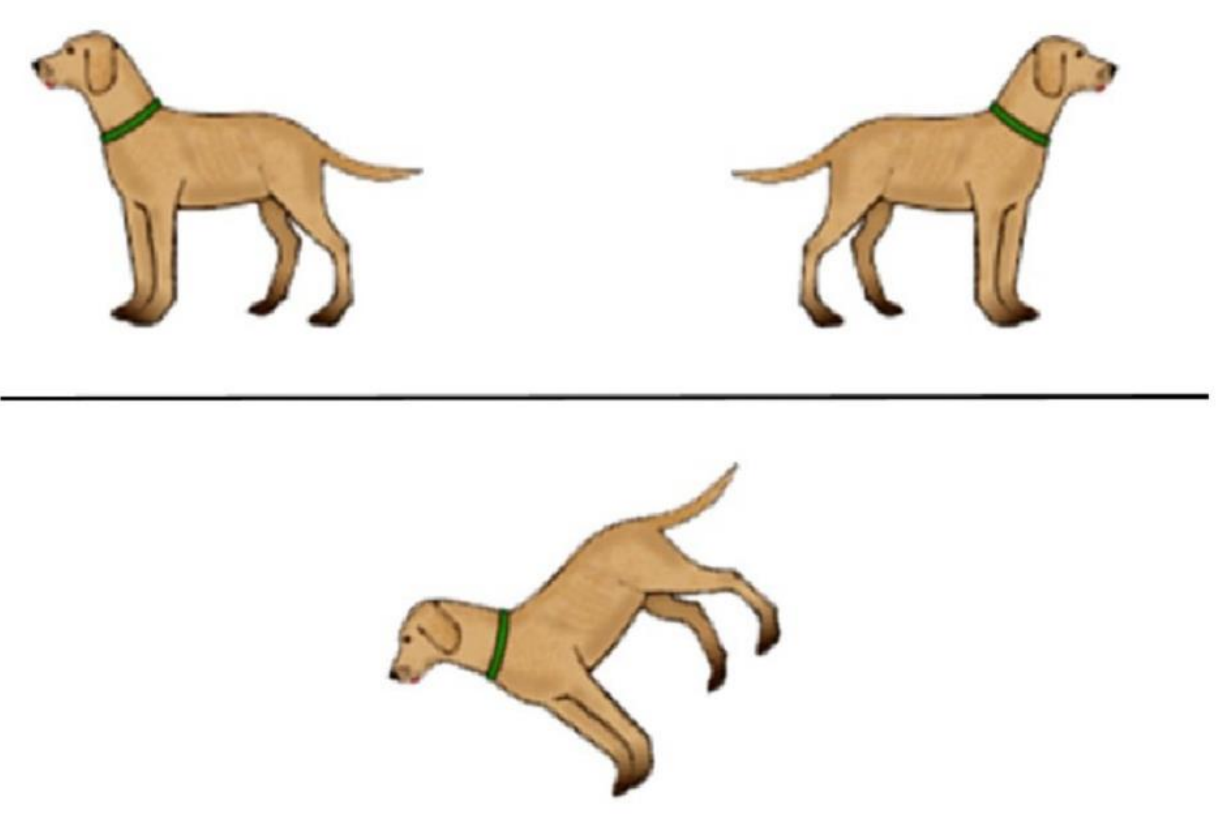

Figure 2: Example stimulus from the mental rotation task (45anti-clockwise trial)

\subsubsection{Image scanning}

The image scanning task was adapted from Wimmer et al. (2016). On 4 practise trials, participants were shown a map of a park where they watched 'Percy the Pirate Parrot' walk between landmarks. Participants had to close their eyes and imagine the parrot walking between two specified landmarks after the experimenter said 'Start'. Participants were instructed to say 'Stop' when they imagined the parrot reaching the second landmark and were then asked how this compared to the Parrot walking across the screen and given further instruction if necessary.

Next participants viewed a map of an island containing a Lighthouse, Volcano, Hut, Pond and Tree for 45 seconds (see Figure 3). The signposts included in Wimmer et al.'s (2016) map of the island were removed as we did not include research questions regarding influence of conceptual knowledge. Following this the landmarks disappeared leaving an empty island. Participants recalled each landmark and then used the mouse to click on the location of each landmark, the landmark then appeared in the correct location and participants could compare their prediction. The island then disappeared, and the participants were instructed to close their eyes and imagine the map of the island. The experimenter read a list of 5 pairs one at a time in the following order: Lighthouse-Tree (262mm), Lighthouse-Volcano (81 mm), Hut-Pond (100mm), Hut-Lighthouse (70mm), Lighthouse-Pond (154mm). As in the practise, participants were instructed to imagine the Parrot walking from the first landmark to the second landmark after the experimenter said 'Start' and for the participant to say 'Stop' when they imagined the parrot reaching the 
second landmark. This was recorded by an experimenter click at 'Start' and an experimenter click at the participants' 'Stop' command. The experimenter response method replicates that of previous experiments which have found no significant difference between experimenter and participant response (Wimmer et al., 2015, 2016).

Finally, participants completed perception control trials whereby the map was visible on the screen while the list of pairs of landmarks was tested. This was to ensure participants were able to perceptually differentiate between the varying differences. Participants were instructed to trace their eyes between the landmarks where the parrot would be walking. For both $\mathrm{Ml}$ and perceptual control conditions, RT was measured as the dependent variable as above.

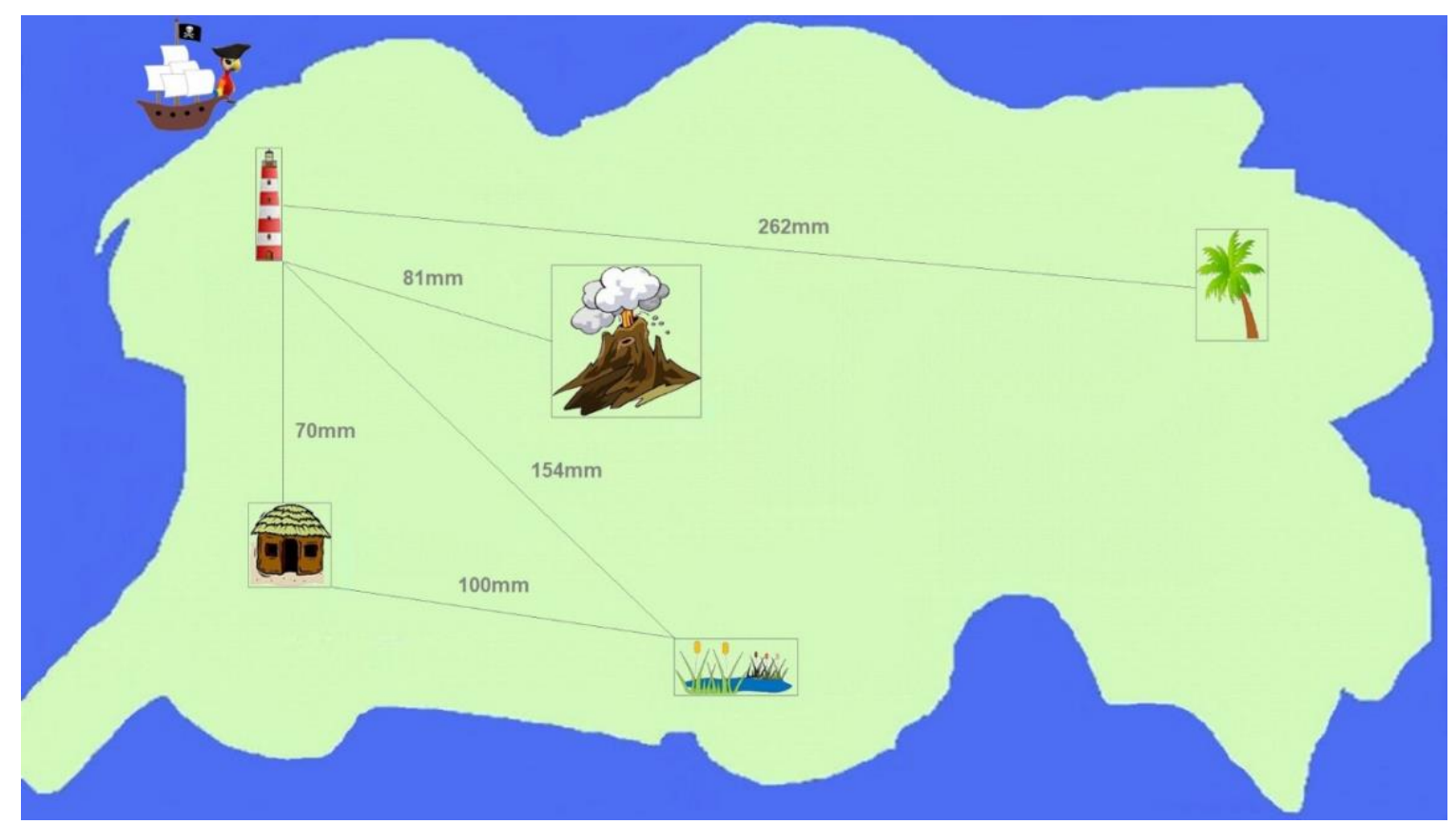

Figure 3: Island scanning task with distances: Hut-Lighthouse $(70 \mathrm{~mm})$, Lighthouse-Volcano (81 mm), Hut-Pond (100mm), Lighthouse-Pond (154mm), Lighthouse-Tree $(262 \mathrm{~mm})$. Lines between landmarks and distance labels added for reference and not presented during task.

\subsubsection{VWM}

Forward span and backward span VWM tasks were administered with the forward span task proceeding the backward span task, in line with standard procedures (Simone et al., 2015). Participants were presented with a $3 \times 3$ grid of lily pads (see Figure 4). To practise, participants completed 3 trials with a sequence of 2 . If participants clicked outside of the lily pad or were incorrect, the practise ended and was repeated. In forward span experimental trials, participants watched a frog appear on multiple lily pads ranging from 2-9. They then were required to click on the lily pads first to last. Each sequence length was repeated 4 times, and sequence length increased by one in each new block. If participants made two or 
more errors in one block, the task stopped. The backward span task followed the same procedure including practise trials, except participants had to click on the lily pads back to front, i.e., in the reverse order. The dependent variable recorded from each task was the maximum sequence length reached (range 2-9).

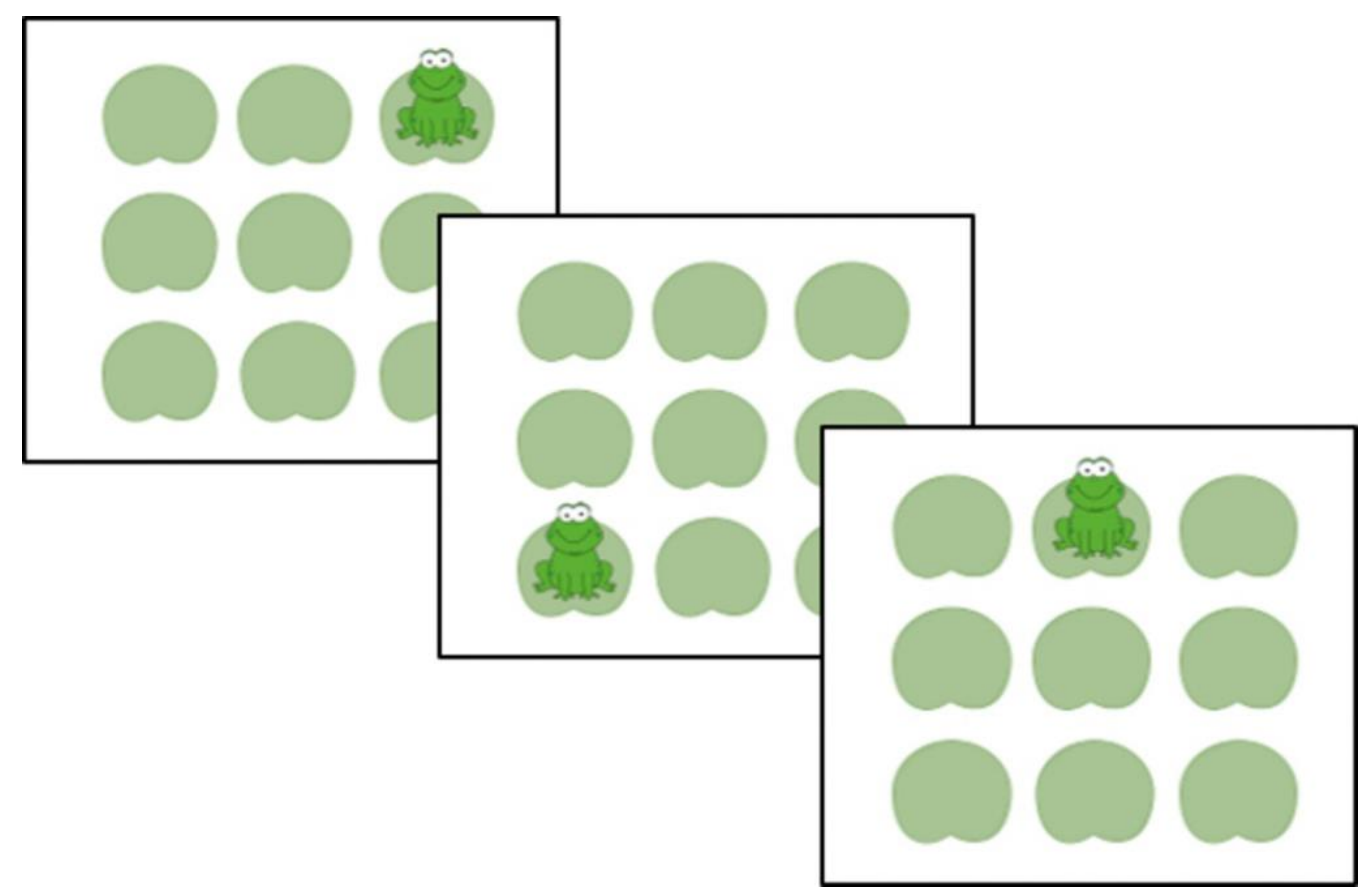

Figure 4: VWM - example sequence from 3 span trial

\subsection{Analysis strategy}

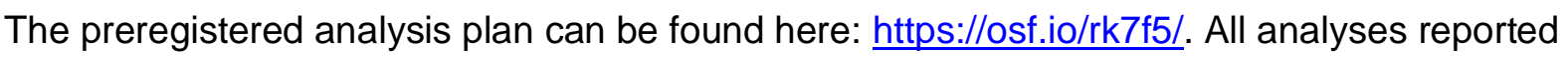
in this manuscript and supplementary materials are detailed in the pre-registration document. Analyses investigating the developmental progression of visual precision of mental images in image generation and image maintenance, mental rotation accuracy and the ability to internally represent distance in image scanning are reported in the supplementary materials. The preregistration includes a correlation matrix per age group based on Wimmer et al.'s (2017) effect sizes (mean $r=.56$ ), which suggested a sample size of 22 per group. However, the coefficients were much smaller than expected for each group (mean r for 6-7-year-olds: -.014; 8-9-year-olds: .012; 10-11-year-olds: .023). Given that our a priori smallest effect size $(r=.56)$ of interest was based on one paper, we wanted to fully establish whether these effects should be considered non-significant and whether we were risking type II errors. Thus, in addition to the correlations per age group (reported in the supplementary materials), we also conducted one correlation matrix controlling for age in years and collapsed across the three groups of primary aged children and we conducted a second correlation matrix with adults. These were powered to detect small to medium effect 
sizes. Tests of normality revealed that most variables were not normally distributed (Kolmogorov-Smirnov Test, ps<.05), therefore, non-parametric Spearman correlation matrices were conducted. To correct for multiple comparisons, $p$ values were Bonferroni corrected (alpha level $0.05 / 15$ comparisons) resulting in $p=.003$. Analyses of slopes of best fitting lines on mental rotation and image scanning RTs are additional to the preregistration, as well as the ANOVA on perception control RT data reported in the supplementary materials. As there are unequal numbers of participants across groups, Welch's F is reported for one-way ANOVAs and Games-Howell correction is applied to posthoc comparisons. As we are comparing adult and child samples in the same analyses, some variance ratios for RT variables exceeded that appropriate for assumptions of parametric tests. Levene's test of equality of variance was violated for the RT dependent variables in all tasks. Given that the sample sizes in each age group are unequal, RT variables were log transformed in order to meet the assumption of normality in multivariate tests. Levene's tests were no longer significant following log transformations of RTs ( $p s>.05$ ). Finally, we had no pre-registered hypotheses regarding gender, however, for completeness, we have checked and ruled out gender effects for all measures ( $p$ 's $>.05$ ).

Cronbach's alpha was conducted to assess the internal consistency of novel measures: image generation, image maintenance and the image scanning imaged distance ratios. The structure of the scores for items in the image generation and image maintenance tasks (i.e., possible scores per $=0,1,2$ ) results in low standard deviations per item on account of the small range of possible scores. Because of this, interitem covariance is not meaningful and it is not appropriate to conduct Cronbach's alpha reliability analyse (Cronbach's alpha uses mean interitem covariance) on the image generation and image maintenance accuracy measures (Cortina, 1993). Thus, for image generation and image maintenance tasks, internal consistency is calculated using RT. Internal consistency analysis was conducted on the child data and the adult data respectively. This revealed good internal consistency for both children (image generation $\alpha=.75$, SD = .76; image maintenance $\alpha=$ $.69, \mathrm{SD}=.71$ ) and adults (image generation $\alpha=.62$, SD $=.62$, image maintenance $\alpha=.56$, $\mathrm{SD}=.65)$, albeit with slightly lower internal consistency in adults. Finally, good internal consistency was also revealed for the Image Scanning imaged distance ratios for both children $(\alpha=.69, \mathrm{SD}=.75)$ and adults $(\alpha=.78, \mathrm{SD}=.83)$. With regard to the reliability of the previously used measures, the VWM measures have been used in previous research with over 300 children and found to have good test-retest reliability (Morris, 2020). The mental rotation task has also been used in a number of previous studies (Bates et al., 2021; Gilligan et al., 2019; Neuburger, Jansen, Heil, \& Quaiser-Pohl, 2011) and is a well-established paradigm. 


\section{Results}

\subsection{Developmental progression of each component of MI}

\subsubsection{Image generation}

A one-way ANOVA examining age group differences in overall accuracy in Image Generation showed a significant main effect of age (Welch's $F(3,70.51)=6.86, p<.001, \eta^{2}=$ $.12)$, whereby post-hoc comparisons show a significant improvement between children aged 6-7 years $(M=13.00, S D=3.25)$ and 10-11-year-olds $(M=15.97, S D=3.19 ; p=.002)$ and between 6-7-year-olds and adults only $(M=15.97, S D=3.52 ; p<.01)$, but no difference across groups from age 8-9-years $(M=14.19, S D=3.20$ ) onwards ( $p>.05$ for all) (Figure $5)$. Further analysis of the visual precision of mental images generated in this task are reported in the supplementary materials (section S1).

\subsubsection{Image maintenance}

A one-way ANOVA investigating age group differences in overall accuracy in image maintenance revealed a significant main effect (Welch's $F(3,66.07)=11.49, p<.001, \eta^{2}=$ $.21)$, whereby there were significant improvements between 6 - to 7-year-olds $(M=14.35, S D$ $=3.61$ ) and the oldest children (10- to 11-year-olds: $M=16.54, S D=2.67 ; p=.037$ ) and 6to 7 -year-olds and adults $(M=17.91, S D=2.57 ; p<.001)$. There was also a significant improvement between 8 - to 9 -year-olds $(M=14.89, S D=3.08)$ and adults $(p<.001$; all other $p s>.05$ ) (Figure 5). Further analysis of the visual precision of mental images maintained in this task are reported in the supplementary materials (section S1).
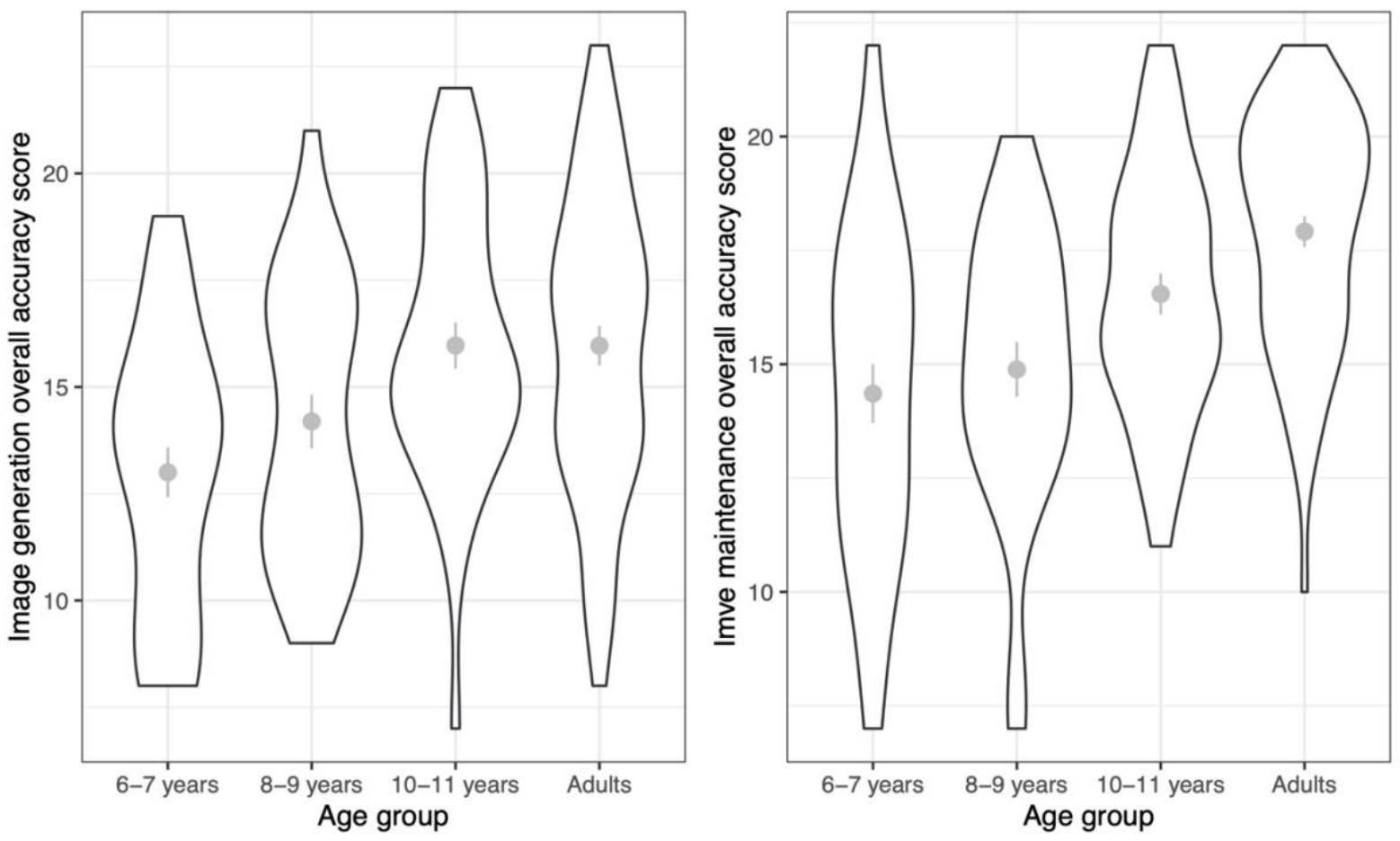
Figure 5: Violin plot showing mean and SE with individual variability for image generation overall accuracy (left) and image maintenance overall accuracy (right)

\subsubsection{Mental rotation}

A mixed ANOVA was conducted on the mean logRT of correct trials per degree of rotation $\left(0^{\circ}, 45^{\circ}, 90^{\circ}, 135^{\circ}\right.$ and $\left.180^{\circ}\right)$ by age group. There was a significant main effect of age $\left(F(3,146)=69.54, p<.001, \eta_{p}^{2}=.58\right)$ on account of significantly longer RTs between each age group and adults, as well as longer RTs in 6- to 7-year-olds compared to 10- to 11year-olds and in 8- to 9-year-olds compared to 10- to 11-year-olds (all $p s<.001$ ). There was a main effect of degree of rotation, which was best explained by a significant linear contrast $\left(F(1,146)=282.05, p<.001, \eta_{p}^{2}=.66\right)$ demonstrating that as degree of rotation increased, $\log R T$ increased. There was also a significant interaction between degree of rotation and age group $\left(F(9.97,485.04)=1.92, p<.05, \eta_{p}^{2}=.04\right)$. Follow up analyses were conducted on the main effect of degree of rotation, for each age group. As can be seen in Figure 6, this revealed that the source of the interaction was a slightly different effect of degrees of rotation for the youngest age group compared to the other groups. The youngest age group showed a significant linear contrast $\left(F\left(1,30=23.42, p<.001, \eta_{p}^{2}=.44\right)\right.$ and significant quadratic contrast $\left(F(1,30)=8.99, p=.005, \eta_{p}^{2}=.23\right)$, on account of an asymptote in performance for higher degrees of rotation. However, note that the linear contrast had the largest $F$ and effect size. All other age groups showed only significant linear contrasts $(p s<.001)$. Mean and SDs of the raw RTs for each degree of rotation is reported in the supplementary materials (Table S2). 


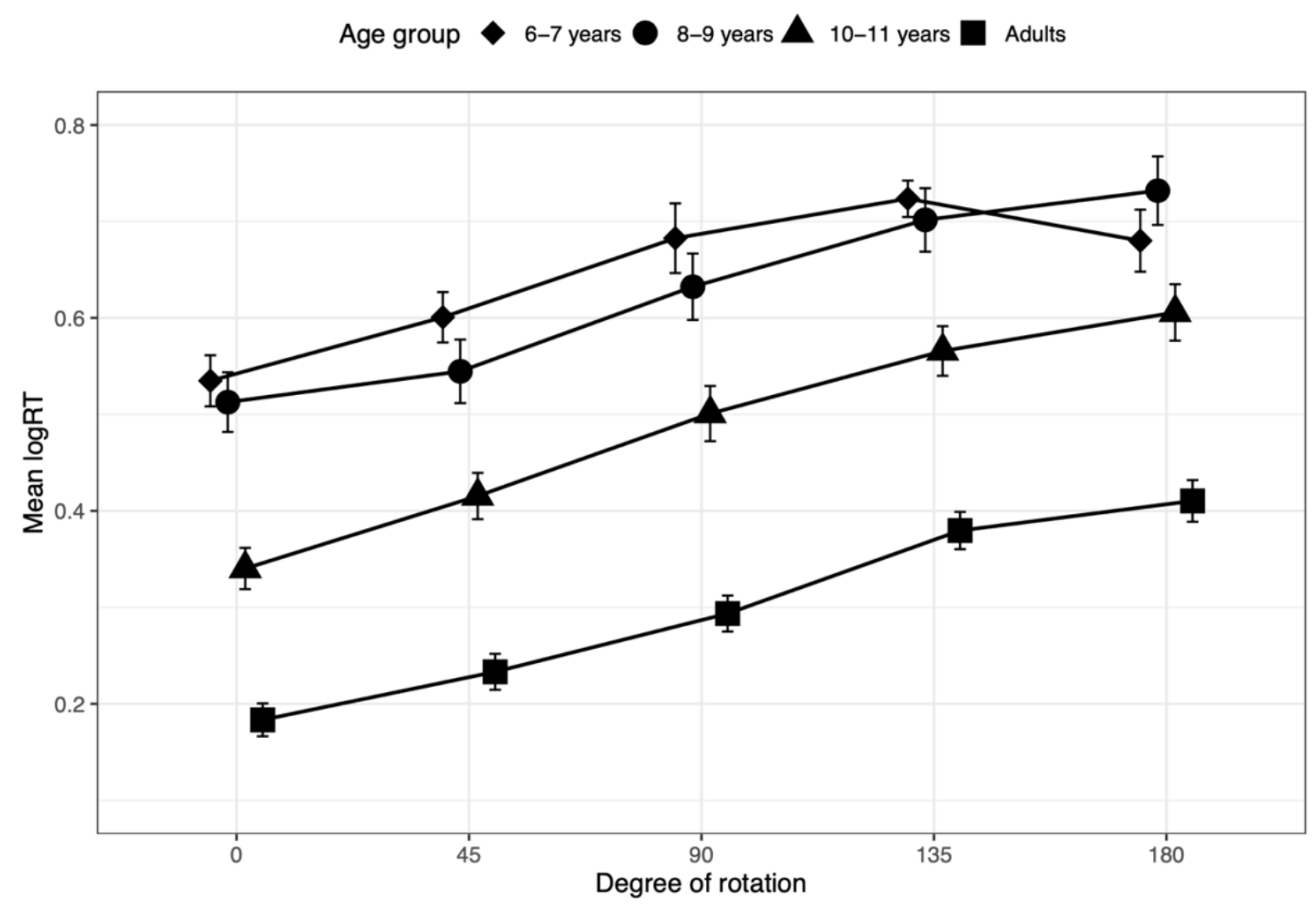

Figure 6: Mean and SE logRT per degree of rotation for each age group in the Mental Rotation task.

In addition to the traditional analyses of mental rotation above, lines of best-fit were calculated to determine the slope value, i.e. the gradient of the line, of mean correct RT across the five degrees of rotation $\left(0^{\circ}, 45^{\circ}, 90^{\circ}, 135^{\circ}, 180^{\circ}\right)$ for each participant. This was calculated as it has been argued the slope values capture individual differences in the speed of the mental rotation operation (Karádi et al., 2001; Kosslyn et al., 1984). A one-way ANOVA of slope values by age group revealed a significant main effect of age group (Welch's $\left.F(3,24.88)=5.52, p=.002, \eta_{p}^{2}=.08\right)$. Post hoc comparisons revealed a significantly steeper slope between 8 - to 9-year-olds $(M=.64, S D=.61)$ and adults $(M=$ $0.31, S D=.23)(p=.005)$. There were no other between group differences (6- to 7-yearolds: $M=.43, S D=.52 ; 10$ - to 11 -year-olds: $M=.53, S D=.36$; all $p s>.05$ ). Analysis of developmental progression of mental rotation accuracy are reported in the supplementary materials.

\subsubsection{Image scanning}

A mixed ANOVA of mean logRT with factors of distance $(70 \mathrm{~mm}, 81 \mathrm{~mm}, 100 \mathrm{~mm}$, $154 \mathrm{~mm}, 262 \mathrm{~mm}$ ) and age group revealed a significant main effect of distance $\left(F(3.36,490.06)=116.57, p<.001, \eta_{p}^{2}=.44\right)$ showing that as distance increased, $\log R T$ 
increased (see Figure 7). Here we report pairwise comparisons rather than contrasts given that the distances are not equally spaced. Pairwise comparisons revealed no significant differences between $70 \mathrm{~mm}, 81 \mathrm{~mm}$ and $100 \mathrm{~mm}$ (ps>.05). This might be expected as the 3 shortest distances are very comparatively close together with differences of only $11 \mathrm{~mm}$ $19 \mathrm{~mm}$ between the landmarks. In turn, there were significant increases in RT between each smaller distance and the two largest distances, as well as a significant increase between the $2^{\text {nd }}$ largest and largest distance (all $\left.p s<.001\right)$. A significant main effect of age group $(F(3,146)$ $\left.=2.751, p<.05, \eta_{p}^{2}=.05\right)$ with a small effect size indicated that 10 - to 11-year-olds had significantly longer responses than adults ( $p<.05$; all other comparisons: $p>.05)$. There was a significant interaction between distance and age group $(F(10.07,490.06)=4.20, p<$ $\left..001, \eta_{p}^{2}=.08\right)$, also with a small effect size. Follow-up analyses showed only adults had the exact pattern of RT differences outlined above (all $p s<.001$ ). Children ages 10-11 years showed almost the same pattern as adults, however there was no significant increase in RT between the $2^{\text {nd }}$ furthest distance and the furthest distance $(154 \mathrm{~mm}<262 \mathrm{~mm} ; p=.116)$. Children age 6-7 and 8-9 years showed significant increases between the two shortest distances and two furthest distances only ( $p s<.05)$ (Figure 7). Mean and SDs of the raw $\mathrm{RT}$ s for each distance are reported in the supplementary materials (Table S3A), alongside the equivalent mixed ANOVA for perception control trials (section S3).

In line with previous methods (Wimmer et al., 2016), slope values were calculated for each participant on non-transformed RT and a one-way ANOVA examining age group differences in slopes was conducted. There was no main effect of age group (Welch's $\left.F(3,62.07)=1.65, p=.186, \eta_{p}^{2}=.02\right)$. 


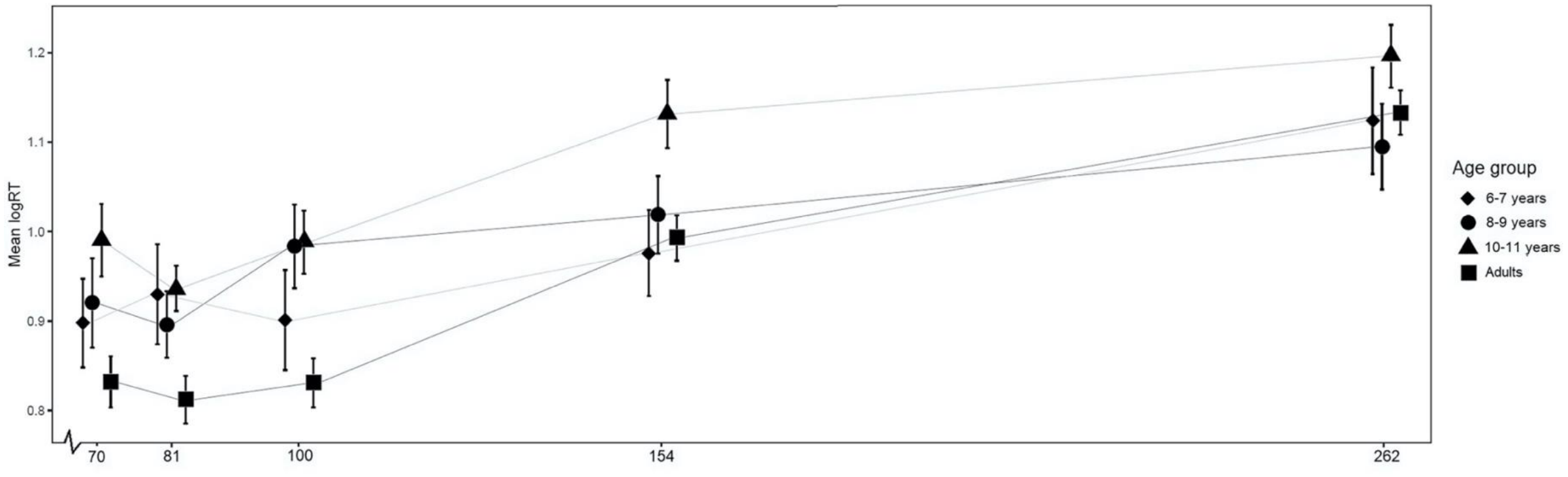

Figure 7: Mean and SE logRT per distance for each age group in the Image Scanning task, $\mathrm{x}$ axis distances to scale 


\subsection{The relationship between the components of MI and VWM}

The following dependent variables, one for each component of $\mathrm{Ml}$, were included in the correlation analyses: overall accuracy in image generation, overall accuracy image maintenance, mean correct RT in mental rotation, mean RT in image scanning. The VWM variables were VWM maintenance (max sequence length/span in the forward VWM task) and VWM manipulation (max sequence length/span in the backward VWM task). As most variables were not normally distributed, Spearman's correlations were conducted. For the child data, we conducted a Spearman correlation matrix on the residuals of the ranks of the variables (age partialled out). A separate Spearman's correlation matrix was conducted with the adult group. Spearman's correlation matrices per age group are reported in the supplementary materials for transparency. Note that the relationships did not differ from the results reported below.

As outlined in Table 2, after correction for multiple comparisons, in children aged 611 years, there were no significant associations between any of the components of MI, suggesting support for a separable component model ( $p s>.003$ ). Additionally, there were no relationships between either VWM maintenance or VWM manipulation and each of the MI components ( $p s>$.003). The same correlation matrix was conducted with the slope values as the mental rotation and image scanning variables, but the findings were the same and there were no significant associations ( $p s>.003$ ).

Table 2

Spearman's correlations on the residuals on the ranks of the variables (age partialled out) between MI measures and VWM maintenance and VWM manipulation in children age 6-11 years $(N=92)$

1. $2 . \quad 3 . \quad 4 . \quad 5 . \quad 6$.

\begin{tabular}{llrrrrrr}
\hline 1. Image generation & - & & & & \\
2. Image maintenance & .242 & - & & & \\
3. Mental rotation & -.043 & -.200 & - & & \\
4. Image scanning & -.154 & -.064 & .001 & - & \\
5. VWM maintenance & -.036 & .112 & -.049 & .080 & - & \\
6. VWM manipulation & .128 & .009 & -.136 & -.023 & .225 & - \\
${ }^{* \star} p<.003$ & & & & &
\end{tabular}

We next investigated how these abilities are related in adulthood (Table 3). These findings were different to those in primary school children. After correcting for multiple comparisons, there was a moderate negative correlation between image maintenance and 
mental rotation $\mathrm{RT}\left(r_{s}=-.487, p<.003\right)$, suggesting higher accuracy in image maintenance is associated with faster correct RT in mental rotation. There were no relationships between measures of $\mathrm{MI}$ and VWM in adults. Finally, there was a positive association between the maintenance and manipulation measures of VWM in adulthood $\left(r_{s}=.463, p<.003\right)$. This correlation matrix was conducted again with the mental rotation and image scanning slopes values. In this analysis, the relationship between image maintenance and mental rotation was no longer present $\left(r_{s}=-.056, p=.676\right)$. All other relationships remained the same.

Table 3

Spearman's correlations between MI measures and maintenance and manipulation in VWM in adults $(\mathrm{N}=57)$

1.

2.

3.

4.

5.

6.

1. Image generation

2. Image maintenance .346

3. Mental rotation $-.219-.487^{* * *}-$

4. Image scanning .171 $-.055$ .073

5. VWM maintenance .007 .301

6. VWM manipulation

.264

$.275 \quad-.299$ $-.075$

${ }^{\star * \star} p<.003$

\section{Discussion}

Here we applied a novel battery of MI tasks to examine the developmental progression of $\mathrm{MI}$ using a novel battery of tasks and to investigate the relationship between the components of $\mathrm{MI}$ and how $\mathrm{MI}$ and VWM are associated with one another throughout childhood and in adulthood. Image generation appears to develop up to age 8-9 years, whereas image maintenance ability develops into later childhood up to 10-11 years. Findings in the mental rotation and image scanning tasks support previous evidence in that RT increased as a function of degree of rotation (mental rotation task) and as a function of distance (image scanning task) from age 6 years. We found evidence for a separablecomponent model of $\mathrm{MI}$ in that there were no associations between components of $\mathrm{MI}$. Components appear to become more integrated in adulthood; demonstrated by a significant negative association between image maintenance and mental rotation in the adult group. Secondly, we found no significant associations between MI and VWM abilities in children or in adults, contrary to expectations.

\subsection{Developmental progression of the components of MI}


Our findings extend previous knowledge regarding the developmental progression of each of the components of $\mathrm{MI}$ by recruiting tasks that allow for the investigation of the visual precision of mental images. Further analyses, presented in the supplementary material, also demonstrated that the generation and maintenance of highly precise mental images from age 6 years. It was also demonstrated that overall accuracy in image generation improves up to 8-9 years whereas as image maintenance appears to improve up to 10-11 years. The latter finding is later than shown in Wimmer et al. (2015). This is likely dependent on the fact that the image maintenance task presented here requires a visual image of high precision to score highly. The use of abstract shapes in the current image generation and image maintenance tasks means that it is more difficult to score highly if individuals are relying on strategies other than MI, such as verbalisation, as the stimuli are not easy to label verbally. Thus, it may be that the ability to maintain a highly precise visual image is still developing into later childhood, whereas the ability to remember locations of known objects, as in Wimmer et al. (2015), requires a less visually precise image and reaches adult-like levels earlier in childhood. Support for this suggestion comes from research investigating development of precision in VWM maintenance. In a study involving 90 males age 7-13 years, researchers presented an orientated, coloured bar, which participants were required to memorise, hold in mind $(500 \mathrm{~ms})$ and had to subsequently rotate a dial to move a probe stimulus to the position of the remembered stimulus. Varying degrees of distance from the target stimulus allowed for measurement of precision of the maintained stimulus. It was found that precision in VWM maintenance continues to develop between age 7 and 13 years (Burnett Heyes et al., 2012). Our findings, which indicate later development of image maintenance in our task that requires visual images of high precision, is in line with the suggestion that the precision at which visual information is held in mind is developing throughout childhood beyond the age range tested in the current study. These novel image generation and image maintenance tasks therefore present a unique opportunity to examine the visual precision of mental images generated and maintained throughout development.

Evidence of a linear time-angle effect in mental rotation and a linear time-distance effect in image scanning in all age groups is in line with previous findings that children from age 4 demonstrate evidence of image transformations and shifting attention across an image (Estes, 1998; Frick et al., 2014; Möhring et al., 2016; Wimmer et al., 2016, 2017). Analysis of the slope values in mental rotation showed a steeper slope in the 8- to 9-year-old group compared to adults, it is currently unclear whether this effect might be attributed to the particular sample of 8-9-year-olds. This could be determined via a replication study. In line with Wimmer et al. (2016), we found no effect of age on steepness of slope on image scanning, suggesting that the ability to shift attention across an image is present from early 
childhood. It is notable that the analyses of mean $\log R T$ for mental rotation and image scanning show a slightly different pattern of results compared to the analyses of RT slopes. This is likely dependent on the suggestion that slopes specifically capture the incremental speed of the transformation (e.g., Kosslyn et al., 1984). Findings regarding the visual precision of $\mathrm{MI}$ throughout development were extended by demonstrating that individuals from age 6 appear to underestimate internally represented distances in mind during image scanning and this underestimation significant increases with increasing distance (analyses reported in supplementary materials). This is in line with findings in visual perception which positive greater underestimation of distance as distance increases (Fukusima et al., 1997; Norman et al., 2016). Thus, it appears that individuals of all ages make similar errors in internally representing distance in the absence of sensory stimuli as they do in distance perception. Importantly, while we can make inferences about development, this initial study involves a cross-sectional sample and longitudinal research is required to provide clarity to developmental findings.

\subsection{Evidence for a separable-component model of MI}

While we expected visual components of $\mathrm{Ml}$ to be related (image generation and image maintenance) and components of $\mathrm{Ml}$ involving transformations (mental rotation and image scanning) to be related, evidence for a separable-component model of $\mathrm{MI}$ was supported. There was some indication of a weak positive correlation between image generation and image maintenance in the adult group and the childhood sample, however, this did not reach significance following correction for multiple comparisons. It might be argued that the correlation between image generation and image maintenance is masked in the childhood sample because the image generation task requires switching to a distractor task in the trial, which might involve other developing cognitive processes such as switching and inhibition. However, our data do not support this assertion because there were no significant differences in performance between image generation and image maintenance between the two tasks in children (if switching had impacted performance, performance would have been poorer for image generation than image maintenance). It is also important to note that there were also no significant associations found between the components of $\mathrm{MI}$ in any of the separate age groups (reported in supplementary materials, section S5), suggesting that components of MI develop separably throughout childhood. Nevertheless, given the limited sample sizes of the individual age groups to detect small coefficients, further research is required with individual age groups with sufficient sample sizes to establish how the relationship between components of MI might differentiate throughout childhood. Future research into the development of MI might benefit from a large sample 
size and a larger battery of tasks in order to conduct a latent variable approach to fully examine the underlying constructs of the component model of $\mathrm{MI}$.

Furthermore, in the adult group, image maintenance accuracy and mental rotation RT are significantly negatively correlated suggesting that as accuracy in Image Maintenance increases, RT in correct trials of mental rotation decreases. This suggests that holding an image in mind is important for both sub-components of MI. However, it should be noted that there was no significant relationship between the mental rotation slope values and image maintenance accuracy. It could be argued that the slope values are more sensitive to transformation of representations, whereas correct trial RT is more sensitive to holding a representation in mind, hence the significant association with image maintenance. The childhood data in the current study supports the theoretical conceptualisation of a distinction between visual (or static) imagery and the ability to transform images (Piaget \& Inhelder, 1971), as well as findings in Aphantasia suggesting typical mental rotation abilities alongside an inability to experience visual imagery (Pounder et al., 2021), however the adult data is not so clear cut. Clearly more research is required. Nonetheless, the suggestion that components of $\mathrm{MI}$ in adulthood are not entirely separable is not new. Specifically, a seminal paper investigating how activation in different areas of the brain predicted performance in different imagery tasks (image generation, image inspection, image transformation, image resolution) found that whilst there was evidence to suggest differential activation between imagery tasks, there was also some evidence for shared activation between image generation, image inspection (which involves maintaining an image) and image transformation in the occipito-parietal sulcus, medial frontal cortex and the early visual areas (Kosslyn et al., 2004). Thus, we tentatively conclude, based on the association between image maintenance and Mental Rotation that visual and spatial aspects of $\mathrm{Ml}$ are at least partially integrated in adults.

\subsection{MI and VWM abilities appear to be unrelated in childhood and adulthood}

This study is the first, to our knowledge, to explicitly compare components of MI with maintenance (forward span) and manipulation (backward span) in VWM in both children and adults. Firstly, the positive association between forward and backward VWM span in adults suggests they are not entirely separable in line with a recent review (Donolato et al., 2017). The evidence presented in our study suggests the components of $\mathrm{MI}$ and maintenance and manipulation in VWM are dissociable in childhood and adulthood. This is surprising given the evidence in adult research demonstrating a relationship between $\mathrm{MI}$ and VWM, even leading some to argue that they are not distinguishable (Tong, 2013). While we do not claim that components of MI and VWM are dissociated based on this data, the findings do lend support 
to the argument that there are individual differences in the types of strategies employed in VWM tasks.

Research determining children's strategies in VWM is limited. If strategies are investigated, the relationship tends to be examined in the opposite direction, i.e. how can different strategy conditions influence VWM and how does this transfer to other abilities, such as mathematics (e.g. Cragg et al., 2017; Swanson, 2015). Studies whereby children are asked to reflect on their strategies are less common. In a study investigating transfer of gains in working memory training, $37 \%$ of the sample of children age $8-11$ years retrospectively reported concentrating harder and $27 \%$ of participants reported a range of other strategies, including rehearsing information and tracing the pattern on the screen with their eyes (Holmes et al., 2009). The variability in participants' responses supports the argument above that a lack of relationship between $\mathrm{MI}$ and VWM measures in children may be dependent on ongoing development of precision of $\mathrm{MI}$, and thus children may be reverting to less efficient strategies to complete VWM maintenance and manipulation tasks. Thus, it may be that while tasks are designed to measure visual WM specifically, there may be variation at the individual level as to whether participants are recruiting MI strategies.

In adult literature, a study introducing irrelevant visual information to try and disrupt performance in VWM found only individuals who scored highly in the sensory strength measure of mental imagery were disrupted (Keogh \& Pearson, 2014), thus implying variance in strategy use during VWM may be dependent on individual differences in MI ability. Moreover, it has been suggested in adult literature that understanding individual variability in strategies and representational format in VWM might explain discrepancies between studies investigating neural mechanisms of VWM (Pearson \& Keogh, 2019; Reeder, 2017). A focus on individual differences in VWM is yet to translate to developmental samples. The findings presented here show that investigating individual differences in how children complete VWM tasks is necessary to determine mechanisms of both VWM and MI development.

In conclusion, using tasks sensitive to the visual precision of MI, generation and maintenance of visually precise mental images appears to develop up to age 8-9 years and 10-11 years, respectively. Children from age 6 years demonstrated the expected effect of increased RT as degree of rotation increased in mental rotation, as well as increased RT as distance increased in image scanning. The data here provides support for a separable component model of $\mathrm{MI}$ in childhood, while image maintenance and mental rotation abilities appear to become related in adulthood. Contrary to expectations, performance in the components of MI was not significantly associated with VWM maintenance or VWM manipulation abilities in childhood or adulthood. Rather than this evidence pointing towards a 
dissociation between $\mathrm{MI}$ and VWM, we have interpreted the findings to suggest that MI may not always be recruited in VWM tasks, especially throughout childhood years where the visual precision of $\mathrm{MI}$ is still developing. Further research should examine the nuances of individual differences in the recruitment of MI strategies in the VWM to establish how MI might support VWM throughout development and in adulthood.

\section{Acknowledgements}

We'd like to thank staff, parents and children at ANONYMISED FOR REVIEW for their contribution to the research. This research is funded by an Economic and Social Research Council PhD Studentship to the first author.

\section{References}

Albers, A. M., Kok, P., Toni, I., Dijkerman, H. C., \& De Lange, F. P. (2013). Shared representations for working memory and mental imagery in early visual cortex. Current Biology, 23(15), 1427-1431. https://doi.org/10.1016/j.cub.2013.05.065

Bates, K. E., Gilligan-Lee, K., \& Farran, E. K. (2020). Reimagining mathematics: The role of mental imagery as a predictor of mathematical calculation skills in childhood. PsyArxiv. https://doi.org/10.31234/osf.io/r7gnc

Bates, K. E., Gilligan-Lee, K., \& Farran, E. K. (2021). Reimagining Mathematics: The Role of Mental Imagery in Explaining Mathematical Calculation Skills in Childhood. Mind, Brain, and Education. https://doi.org/10.1111/mbe.12281

Berg, D. H. (2008). Working memory and arithmetic calculation in children: The contributory roles of processing speed, short-term memory, and reading. Journal of Experimental Child Psychology, 99(4), 288-308. https://doi.org/10.1016/j.jecp.2007.12.002

Blajenkova, O., Kozhevnikov, M., \& Motes, M. A. (2006). Object-spatial imagery: A new selfreport imagery questionnaire. Applied Cognitive Psychology, 20(2), 239-263. https://doi.org/10.1002/acp.1182

Borst, G., \& Kosslyn, S. M. (2010). Individual differences in spatial mental imagery. Quarterly Journal of Experimental Psychology, 63(10), 2031-2050. https://doi.org/10.1080/17470211003802459

Bruner, J. S. (1966). Toward a theory of instruction. Havard University Press.

Bull, R., Espy, K. A., \& Wiebe, S. A. (2008). Short-term memory, working memory, and executive functioning in preschoolers: Longitudinal predictors of mathematical achievement at age 7 years. Developmental Neuropsychology, 33(3), 205-228. 
https://doi.org/10.1080/87565640801982312

Burnett Heyes, S., Zokaei, N., van der Staaij, I., Bays, P. M., \& Husain, M. (2012).

Development of visual working memory precision in childhood. Developmental Science, 15(4), 528-539. https://doi.org/10.1111/j.1467-7687.2012.01148.x

Commodari, E., Guarnera, M., Di Stefano, A., \& Di Nuovo, S. (2020). Children Learn to Read: How Visual Analysis and Mental Imagery Contribute to the Reading Performances at Different Stages of Reading Acquisition. Journal of Psycholinguistic Research, 49(1), 59-72. https://doi.org/10.1007/s10936-019-09671-w

Cortina, J. M. (1993). What Is Coefficient Alpha? An Examination of Theory and Applications. Journal of Applied Psychology, 78(1), 98-104.

https://doi.org/10.1037/0021-9010.78.1.98

Cowan, N. (2001). The magical number 4 in short-term memory: A reconsideration of mental storage capacity. Behavioral and Brain Sciences, 24(1), 87-114.

https://doi.org/10.1017/S0140525X01003922

Cragg, L., Richardson, S., Hubber, P. J., Keeble, S., \& Gilmore, C. (2017). When is working memory important for arithmetic? The impact of strategy and age. PLOS ONE, 12(12), 1-18. https://doi.org/10.1371/journal.pone.0188693

Dijkstra, N., Bosch, S. E., \& van Gerven, M. A. J. (2017). Vividness of Visual Imagery Depends on the Neural Overlap with Perception in Visual Areas. The Journal of Neuroscience, 37(5), 1367-1373. https://doi.org/10.1523/jneurosci.3022-16.2016

Dijkstra, N., Bosch, S. E., \& van Gerven, M. A. J. (2019). Shared Neural Mechanisms of Visual Perception and Imagery. Trends in Cognitive Sciences, 23(5), 423-434. https://doi.org/10.1016/j.tics.2019.02.004

Donolato, E., Giofrè, D., \& Mammarella, I. C. (2017). Differences in verbal and visuospatial forward and backward order recall: A review of the literature. Frontiers in Psychology, 8:663. https://doi.org/10.3389/fpsyg.2017.00663

Estes, D. (1998). Young Children's Awareness of Their Mental Activity: The Case of Mental Rotation. Child Development, 69(5), 1345. https://doi.org/10.2307/1132270

Frick, A., Hansen, M. A., \& Newcombe, N. S. (2013). Development of mental rotation in 3- to 5-year-old children. Cognitive Development, 28(4), 386-399.

https://doi.org/10.1016/j.cogdev.2013.06.002

Frick, A., Möhring, W., \& Newcombe, N. S. (2014). Development of mental transformation 
abilities. Trends in Cognitive Sciences, 18(10), 536-542.

https://doi.org/10.1016/j.tics.2014.05.011

Fukusima, S. S., Loomis, J. M., \& Da Silva, J. A. (1997). Visual Perception of Egocentric Distance as Assessed by Triangulation. Journal of Experimental Psychology: Human Perception and Performance, 23(1), 86-100. https://doi.org/10.1037/0096-1523.23.1.86

Ganis, G. (2013). Visual mental imagery. In Multisensory imagery (pp. 9-28). Springer New York. https://doi.org/10.1007/978-1-4614-5879-1_2

Gilligan, K. A., Thomas, M. S. C., \& Farran, E. K. (2019). First demonstration of effective spatial training for near transfer to spatial performance and far transfer to a range of mathematics skills at 8 years. Developmental Science, 23(4), e12909. https://doi.org/10.1111/desc.12909

Giofrè, D., Mammarella, I. C., Ronconi, L., \& Cornoldi, C. (2013). Visuospatial working memory in intuitive geometry, and in academic achievement in geometry. Learning and Individual Differences, 23(1), 114-122. https://doi.org/10.1016/j.lindif.2012.09.012

Hawes, Z., Sokolowski, H. M., Ononye, C. B., \& Ansari, D. (2019). Neural underpinnings of numerical and spatial cognition: An fMRI meta-analysis of brain regions associated with symbolic number, arithmetic, and mental rotation. Neuroscience and Biobehavioral Reviews, 103(January), 316-336. https://doi.org/10.1016/j.neubiorev.2019.05.007

Hegarty, M., \& Kozhevnikov, M. (1999). Spatial abilities, working memory, and mechanical reasoning. In Visual and Spatial Reasoning in Design. https://doi.org/10.1017/CBO9780511610448.005

Hespos, S. J., \& Rochat, P. (1997). Dynamic mental representation in infancy. Cognition, 64(2), 153-188. https://doi.org/10.1016/S0010-0277(97)00029-2

Holmes, J., Gathercole, S. E., \& Dunning, D. L. (2009). Adaptive training leads to sustained enhancement of poor working memory in children. Developmental Science, 12(4), 915. https://doi.org/10.1111/j.1467-7687.2009.00848.x

Kail, R., Pellegrino, J., \& Carter, P. (1980). Developmental changes in mental rotation. Journal of Experimental Child Psychology, 29(1), 102-116. https://doi.org/10.1016/0022-0965(80)90094-6

Karádi, K., Kállai, J., \& Kovács, B. (2001). Cognitive Subprocesses of Mental Rotation: Why is a Good Rotator Better Than a Poor One? Perceptual and Motor Skills, 93(2), 333337. https://doi.org/10.2466/PMS.2001.93.2.333 
Keogh, R., \& Pearson, J. (2011). Mental Imagery and Visual Working Memory. PLOS ONE, 6(12), e29221. https://doi.org/10.1371/journal.pone.0029221

Keogh, R., \& Pearson, J. (2014). The sensory strength of voluntary visual imagery predicts visual working memory capacity. Journal of Vision, 14:7(12), 1-13. https://doi.org/10.1167/14.12.7.doi

Keogh, R., \& Pearson, J. (2017). The blind mind: No sensory visual imagery in aphantasia. Cortex. https://doi.org/10.1016/J.CORTEX.2017.10.012

Kosslyn, S. M. (1980). Image and Mind. Havard University Press.

Kosslyn, S. M. (1996). Image and brain: the resolution of the imagery debate. MIT Press.

Kosslyn, S. M., Brunn, J., Cave, K. R., \& Wallach, R. W. (1984). Individual differences in mental imagery ability: A computational analysis. Cognition, 18(1-3), 195-243.

Kosslyn, S. M., Ganis, G., \& Thompson, W. L. (2003). Mental imagery: against the nihilistic hypothesis. Trends in Cognitive Sciences, 7(3), 109-111. https://doi.org/10.1016/S1364-6613(03)00021-4

Kosslyn, S. M., \& Thompson, W. L. (2003). When Is Early Visual Cortex Activated During Visual Mental Imagery? Psychological Bulletin, 129(5), 723-746. https://doi.org/10.1037/0033-2909.129.5.723

Kosslyn, S. M., Thompson, W. L., \& Ganis, G. (2006). The case for mental imagery. Oxford University Press.

Kosslyn, S. M., Thompson, W., Shephard, J. M., Ganis, G., Bell, D., Danovitch, J., Wittenberg, L. A., \& Alpert, N. M. (2004). Brain rCBF and performance in visual imagery tasks: Common and distinct processes. European Journal of Cognitive Psychology, 16(5), 696-716. https://doi.org/10.1080/09541440340000475

Leopold, C., Mayer, R., \& Barbara, S. (2015). An Imagination Effect in Learning From Scientific Text. Journal of Educational Psychology, 107(1), 1-17. https://doi.org/10.1037/a0037142

Logie, R. H. (1995). Visuo-spatial working memory. L. Erlbaum Associates.

Martinussen, R., \& Tannock, R. (2006). Working memory impairments in children with attention-deficit hyperactivity disorder with and without comorbid language learning disorders. Journal of Clinical and Experimental Neuropsychology, 28(7), 1073-1094. https://doi.org/10.1080/13803390500205700 
Massonnié, J., Frasseto, P., Mareschal, D., \& Kirkham, N. Z. (2020). Scientific Collaboration with Educators: Practical Insights from an in-Class Noise-Reduction Intervention. Mind, Brain, and Education. https://doi.org/10.1111/mbe.12240

Möhring, W., Newcombe, N. S., \& Frick, A. (2016). Using Mental Transformation Strategies for Spatial Scaling: Evidence From a Discrimination Task. Journal of Experimental Psychology: Learning, Memory, and Cognition, 42(9), 1473-1479. https://doi.org/10.1037/xIm0000240

Moore, D. S., \& Johnson, S. P. (2011). Mental Rotation of Dynamic, Three-Dimensional Stimuli by 3-Month-Old Infants. Infancy, 16(4), 435-445. https://doi.org/10.1111/j.15327078.2010.00058.x

Morris, S. C. (2020). Evaluating achievement on mathematics and science problems: The role of global and local processing. Doctoral Thesis, UCL (University College London). .

Naselaris, T., Olman, C. A., Stansbury, D. E., Ugurbil, K., \& Gallant, J. L. (2015). A voxelwise encoding model for early visual areas decodes mental images of remembered scenes. Neurolmage, 105, 215-228.

https://doi.org/10.1016/J.NEUROIMAGE.2014.10.018

Neuburger, S., Jansen, P., Heil, M., \& Quaiser-pohl, C. (2011). Gender differences in preadolescents' mental-rotation performance: Do they depend on grade and stimulus type? Personality and Individual Differences, 50(8), 1238-1242.

https://doi.org/10.1016/j.paid.2011.02.017

Neuburger, S., Jansen, P., Heil, M., \& Quaiser-Pohl, C. (2011). Gender differences in preadolescents' mental-rotation performance: Do they depend on grade and stimulus type? Personality and Individual Differences, 50(8), 1238-1242.

https://doi.org/10.1016/j.paid.2011.02.017

Norman, J. F., Adkins, O. C., \& Pedersen, L. E. (2016). The visual perception of distance ratios in physical space. Vision Research, 123, 1-7. https://doi.org/10.1016/j.visres.2016.03.009

Pearson, J., Clifford, C., \& Tong, F. (2008). The Functional Impact of Mental Imagery on Conscious Perception. Current Biology, 18(13), 982-986. https://doi.org/10.1016/j.cub.2008.05.048

Pearson, J., \& Keogh, R. (2019). Redefining Visual Working Memory: A Cognitive-Strategy, Brain-Region Approach. Current Directions in Psychological Science, 28(3), 266-273. 
https://doi.org/10.1177/0963721419835210

Pearson, J., \& Kosslyn, S. M. (2015). The heterogeneity of mental representation: Ending the imagery debate. PNAS, 112(33), 10089-10092.

https://doi.org/10.1073/pnas.1504933112

Piaget, J., \& Inhelder, B. (1971). Mental imagery in the child. Basic.

Pounder, Z., Jacob, J., Evans, S., Loveday, C., Eardley, A., \& Silvanto, J. (n.d.). Individuals with congenital aphantasia show no significant neuropsychological deficits on imageryrelated memory tasks. https://doi.org/10.31234/OSF.IO/GQAYT

Pylyshyn, Z. W. (2002). Mental imagery: In search of a theory. Behavioural and Brain Sciences, 25, 157-237. https://doi.org/10.1017/S0140525X02280048

Reeder, R. R. (2017a). Individual differences shape the content of visual representations. Vision Research, 141, 266-281. https://doi.org/10.1016/j.visres.2016.08.008

Reeder, R. R. (2017b). Individual differences shape the content of visual representations. Vision Research, 141, 266-281. https://doi.org/10.1016/j.visres.2016.08.008

Sala, G., \& Gobet, F. (2017). Working memory training in typically developing children: A multilevel meta-analysis. Psychonomic Bulletin and Review, 53(4), 671-685. https://doi.org/10.3758/s13423-019-01681-y

Shepard, R. N., \& Metzler, J. (1971). Mental Rotation of Three-Dimensional Objects. Science, 171, 701-703.

Simmons, F. R., Willis, C., \& Adams, A. M. (2012). Different components of working memory have different relationships with different mathematical skills. Journal of Experimental Child Psychology, 111(2), 139-155. https://doi.org/10.1016/j.jecp.2011.08.011

Simone, A. N., Bédard, A. C. V., Marks, D. J., \& Halperin, J. M. (2015). Good holders, bad shufflers: An examination of working memory processes and modalities in children with and without attention-deficit/hyperactivity disorder. Journal of the International Neuropsychological Society, 22(1), 1-11. https://doi.org/10.1017/S1355617715001010

Swanson, H. L. (2015). Cognitive strategy interventions improve word problem solving and working memory in children with math disabilities. Frontiers in Psychology, 6, 1099. https://doi.org/10.3389/fpsyg.2015.01099

Tong, F. (2013). Imagery and visual working memory: One and the same? Trends in Cognitive Sciences, 17(10), 489-490. https://doi.org/10.1016/j.tics.2013.08.005 
Wimmer, M. C., Maras, K. L., Robinson, E. J., Doherty, M. J., \& Pugeault, N. (2015). How visuo-spatial mental imagery develops: Image generation and maintenance. PLoS ONE, 10(11), 1-20. https://doi.org/10.1371/journal.pone.0142566

Wimmer, M. C., Maras, K. L., Robinson, E. J., \& Thomas, C. (2016). The format of children's mental images: Evidence from mental scanning. Cognition, 154, 49-54. https://doi.org/10.1016/j.cognition.2016.05.017

Wimmer, M. C., Robinson, E. J., \& Doherty, M. J. (2017). Are developments in mental scanning and mental rotation related? PLOS ONE, 12(2), 1-12. https://doi.org/10.1371/journal.pone.0171762

Zeman, A., Dewar, M., \& Della Sala, S. (2015). Lives without imagery - Congenital aphantasia. Cortex, 73, 378-380. https://doi.org/10.1016/j.cortex.2015.05.019 